\title{
High-Speed SiGe BiCMOS Technologies: 120-nm Status and End-of-Roadmap Challenges
}

\author{
P. Chevalier ${ }^{1}$, B. Barbalat ${ }^{1,2}$, M. Laurens ${ }^{1}$, B. Vandelle ${ }^{1}$, L. Rubaldo ${ }^{1}$, B. Geynet ${ }^{1,3}$, \\ S.P. Voinigescu ${ }^{4}$, T.O. Dickson ${ }^{4}$, N. Zerounian ${ }^{2}$, S. Chouteau ${ }^{1}$, D. Dutartre ${ }^{1}$, \\ A. Monroy ${ }^{1}$, F. Aniel ${ }^{2}$, G. Dambrine ${ }^{3}$, and A. Chantre ${ }^{1}$
}

1) STMicroelectronics, Crolles, 850 rue Jean Monnet, F-38926 Crolles, France

2) Institut d'Electronique Fondamentale, Bât 220, Université Paris-Sud 11, 91405 Orsay, France 3) IEMN-DHS, UMR CNRS 8520, USTL, Avenue Poincaré - BP69, 59652 Villeneuve d'Ascq, France 4) Edward S. Rogers Sr. Dept. of ECE, University of Toronto, Toronto, ON M5S 3G4, Canada

\begin{abstract}
This paper presents the status of high-speed SiGe BiCMOS technologies at STMicroelectronics. Process and electrical characteristics of two $120-\mathrm{nm}$ platforms, qualified or under development, are presented together with results demonstrated on optical and millimeter-wave circuits. Advanced developments addressing end-of-roadmap BiCMOS are also presented and discussed.
\end{abstract}

Index Terms - BiCMOS, Heterojunction Bipolar Transistors (HBT), millimeter-wave circuits, optical communications, Silicon Germanium (SiGe).

\section{INTRODUCTION}

SiGe BiCMOS technologies extend the application range of Si technologies towards higher frequencies. Just as the frequency limit of CMOS technologies is increased with gate length reduction, encroaching on markets and products which were covered by BiCMOS in the past, $\mathrm{SiGe} \mathrm{BiCMOS}$ is now able to address applications above $50 \mathrm{GHz}$, previously the domain of III-V technologies. Although these very same high-speed applications are also targeted by advanced CMOS technologies, SiGe BiCMOS continues to have the performance edge [1]. This paper presents the status of ST's most advanced high-speed SiGe BiCMOS technologies going from a production 120-nm BiCMOS platform to current developments on high-speed HBTs for end-of-roadmap CMOS nodes. Approaches to increase device performance, which take into account integration constraints of advanced CMOS, are summarized and results are presented. We finally discuss the perspectives of high-speed BiCMOS technologies.

\section{CURRENT StATUS: 120-NM BiCMOS PlatForMS}

\section{A. A platform available for production: BiCMOS9}

The most advanced high-speed SiGe BiCMOS technology in production at STMicroelectronics is the so- called BiCMOS9 [2]. This technology, whose main characteristics are summarized in Table 1, offers a quasi self-aligned (QSA) 160-GHz $f_{T} / f_{\max } \mathrm{Si} / \mathrm{SiGeC} \operatorname{HBT}\left(f_{T}\right.$ and $f_{\text {max }}$ being respectively the transit and the maximum oscillation frequencies), dual $V_{T}$ (high performance / low leakage) and dual gate oxide (1.2 V/2.5 V) 120-nm CMOS devices, passives and a 6-level copper back-end. A high-voltage $\mathrm{HBT}\left(B V_{C E O}=3 \mathrm{~V}\right)$ is also available.

Table 1 ST 120-nm high-speed SiGe BiCMOS technologies.

\begin{tabular}{|c|c|c|c|}
\hline Technology Targets & BiCMOS9 & \multicolumn{2}{|c|}{ BiCMOS9MW } \\
\hline $\begin{array}{l}\text { CMOS } \\
\text { (NMOS \& PMOS) }\end{array}$ & \multicolumn{3}{|c|}{$\begin{array}{c}\mathrm{T}_{\mathrm{OX}}=20 \mathrm{~nm}(\mathrm{GO} 1) / \mathrm{T}_{\mathrm{OX}}=50 \mathrm{~nm}(\mathrm{GO} 2)- \\
\text { High \& Low } \mathrm{V}_{\mathrm{T}}(\mathrm{mV})\end{array}$} \\
\hline $\mathrm{N}: 120 \mathrm{~nm} / 1.2 \mathrm{~V}(\mathrm{GO} 1)$ & 340 & 450 & 340 \\
\hline P: 120nm/1.2V (GO1) & 300 & 395 & 300 \\
\hline $\mathrm{N}: 280 \mathrm{~nm} / 2.5 \mathrm{~V}(\mathrm{GO} 2)$ & 140 & 430 & 140 \\
\hline $\mathrm{P}: 280 \mathrm{~nm} / 2.5 \mathrm{~V}(\mathrm{GO} 2)$ & 180 & 485 & 180 \\
\hline Si/SiGe:C HBT & $\begin{array}{l}\text { QSA structure } \\
\mathrm{W}_{\mathrm{E}}=170 \mathrm{~nm}\end{array}$ & \multicolumn{2}{|c|}{$\begin{array}{l}\text { FSA structure } \\
\mathrm{W}_{\mathrm{E}}=120 \mathrm{~nm}\end{array}$} \\
\hline$f_{T}(\mathrm{GHz})$ & 160 & \multicolumn{2}{|c|}{230} \\
\hline$f_{\max }(\mathrm{GHz})$ & 160 & \multicolumn{2}{|c|}{280} \\
\hline$B V_{C E O}(\mathrm{~V})$ & 1.8 & \multicolumn{2}{|c|}{1.6} \\
\hline BEOL $(\mathrm{Cu})$ & $6 \mathrm{ML}+\mathrm{Al}$ & \multicolumn{2}{|c|}{$6 \mathrm{ML}+\mathrm{Al}$} \\
\hline thin / thick Cu layers & $5 / 1$ & \multicolumn{2}{|c|}{$3 / 3$} \\
\hline$\alpha @ 80 G H z(\mathrm{~dB} / \mathrm{mm})$ & 1.2 & \multicolumn{2}{|c|}{0.5} \\
\hline
\end{tabular}

Manufacturability of this technology is assessed by the histograms shown in Fig. 1 for the current gain (at medium injection) and the pinched base resistance of bipolar transistors. These statistics have been collected on 45 lots produced in the last 9 months (January $1^{\text {st }}$ to beginning of October 2006). Standard deviation is about $10 \%$ for the current gain and below $5 \%$ for the pinched base resistance.

BiCMOS9 has been designed to address optical networking and wireless applications up to $40 \mathrm{~Gb} / \mathrm{s}-$ $40 \mathrm{GHz}$. Numerous circuits have been fabricated in this technology, demonstrating its suitability for the targeted applications. A low-power flip-flop (2.5 V / $20 \mathrm{~mW}$ ) has been validated at 40+ Gb/s [3]. Fig. 2 and Fig. 3 show the layout and 45-Gb/s eye diagram of this circuit. Circuits 
operating at $80 \mathrm{~Gb} / \mathrm{s}$ have also been demonstrated using the MOS-HBT ECL and CML logic families [4]-[5]. Millimeter-wave building blocks such as a $40-\mathrm{GHz}$ LNA [6], a 70-GHz frequency divider [7] and a $95-\mathrm{GHz} \mathrm{VCO}$ [8] further illustrate the technology capabilities.

The largest circuit in production today has a surface area close to $200 \mathrm{~mm}^{2}$. Yield up to $35 \%$, a record for such a large ASIC, is reached thanks to a $D_{0}$ as low as 110 defects $/ \mathrm{m}^{2}$.

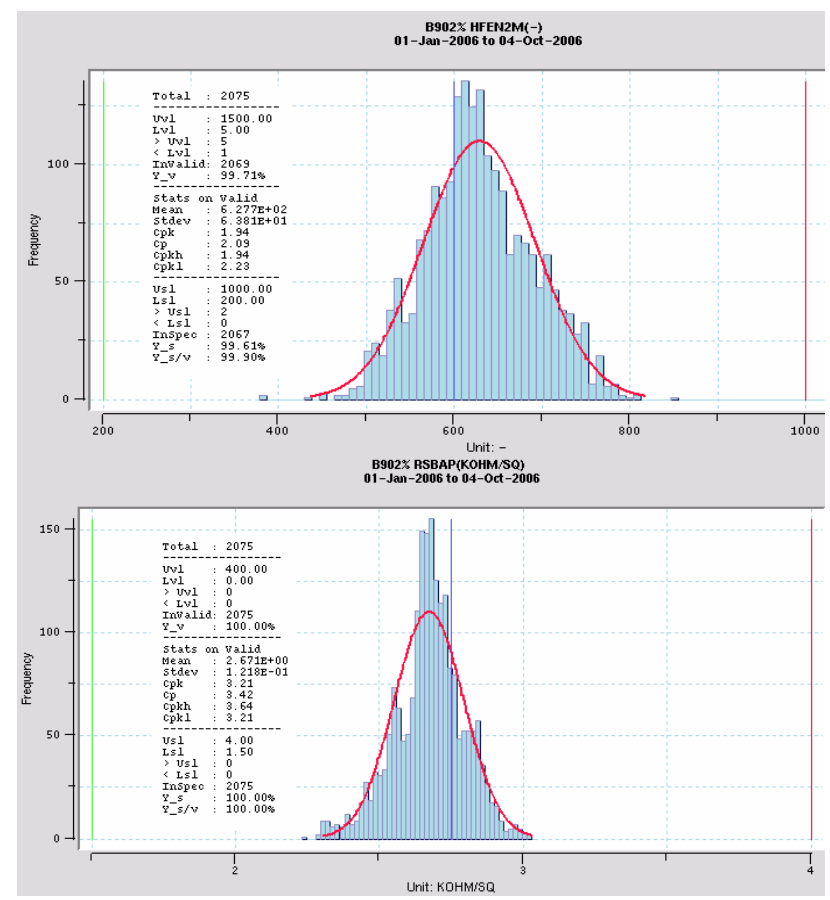

Fig. 1. Current gain at $V_{B E}=0.75 \mathrm{~V}$ of $0.17 \times 5.6 \mu \mathrm{m}^{2} \mathrm{HBTs}$ (Top) and Pinched Base Resistance (Bottom) histograms built with measurements done on 45 BiCMOS9 lots produced over about 9 months.

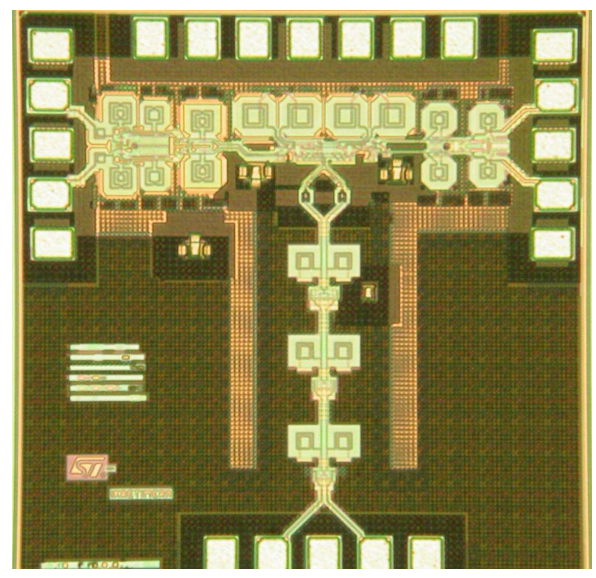

Fig. 2. ST 120-nm BiCMOS9 retimer die photo [3].

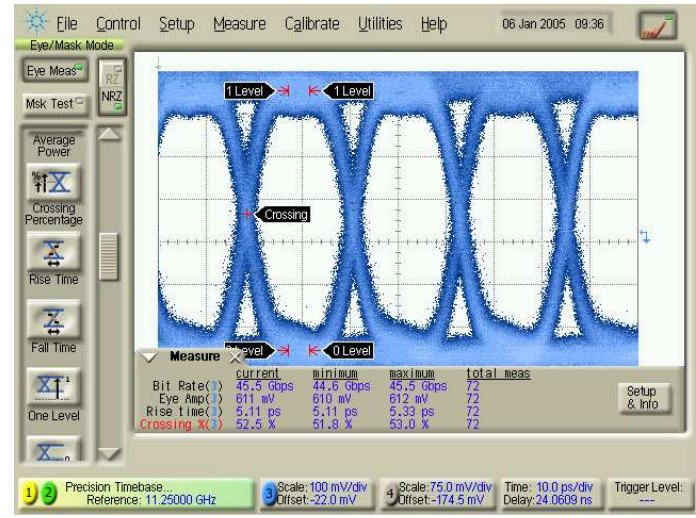

Fig. 3. ST 120-nm BiCMOS9 $45 \mathrm{~Gb} / \mathrm{s}$ output eye diagram [3].

B. A new platform dedicated to millimeter-wave applications: BiCMOS9MW

Applications such as $60-\mathrm{GHz}$ WLAN, 77-GHz automotive radars and $80-\mathrm{Gb} / \mathrm{s}$ optical communications can not be addressed with BiCMOS9 technology. Since a first limitation is the HBT performance, many studies have been conducted to increase $f_{T}$ and $f_{\max }$ above $200 \mathrm{GHz}$ [9][10]. 230-GHz $f_{T}$ and 280-GHz $f_{\max }$ (cf. Fig. 4) have been achieved with a fully self-aligned (FSA) architecture using selective epitaxial growth of the base. The device features record low minimum noise figures $N F_{\min }$ of $1.2 \mathrm{~dB}$ at $40 \mathrm{GHz}$ and $1.6 \mathrm{~dB}$ at $60 \mathrm{GHz}$ (cf. Fig. 4).

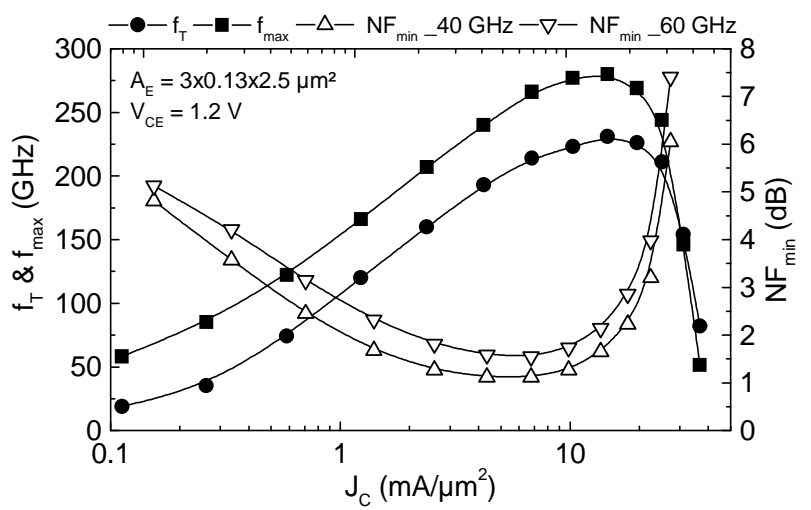

Fig. 4. $f_{T}, f_{\max }$ and $N F_{\min } @ 40 \mathrm{GHz}$ and $60 \mathrm{GHz}$ vs. current density of a $3 \times 0.13 \times 2.5 \mu \mathrm{m}^{2} \mathrm{FSA} \mathrm{Si} / \mathrm{SiGeC} \mathrm{HBT}$.

This FSA architecture has reached a good maturity level. Fig. 5 exhibits indeed the full mapping (35 dice) of Gummel characteristics measured on yield monitors (arrays of more than 3000 devices). None of the measurable device is failing. Furthermore, collector current is ideal down to a $V_{B E}=0 \mathrm{~V}$ and base current remains below $0.2 \mathrm{nA}$ at low injection, i.e. below the $\mathrm{pA} / \mu \mathrm{m}^{2}$ for all the arrays. The shape of the base 
current observed at low $V_{B E}$ on yield monitors is the signature of a band-to-band tunneling current [11]. Besides, Fig. 6 shows that HF characteristics are very uniform with standard deviations below $2 \%$ within a wafer (76 dice) for both $f_{T}$ and $f_{\max }$.

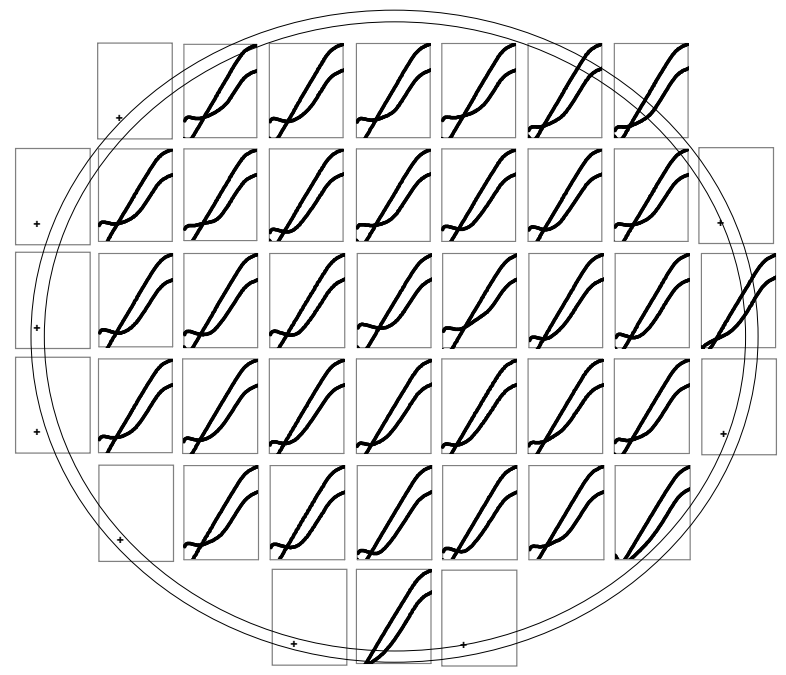

Fig. 5. Mapping of Gummel characteristics at $V_{B C}=0 \mathrm{~V}$ of $240 / 270 \mathrm{GHz} f_{T} / f_{\max } \mathrm{FSA} \mathrm{Si} / \mathrm{SiGeC} \mathrm{HBT}$ arrays $\left(3024 \times 0.13 \times 0.5 \mu \mathrm{m}^{2}\right) . V_{B E}$ scale (lin) is from 0 to $0.9 \mathrm{~V}, I_{C}$ and $I_{B}$ scale $(\log )$ is from $1 \mathrm{pA}$ to $100 \mathrm{~mA}$. ' + ' indicates structure position in the die and notch is at bottom.

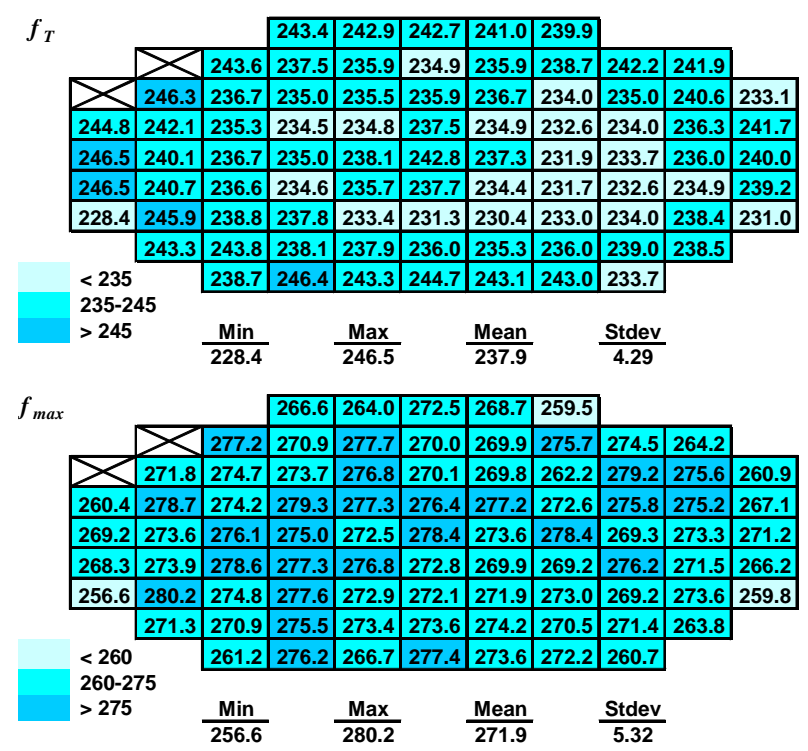

Fig. 6. $f_{T}$ and $f_{\max }$ mappings [GHz] of a $240 / 270 \mathrm{GHz} f_{T} / f_{\max }$ $0.13 \times 3.6 \mu \mathrm{m}^{2}$ FSA HBT at $V_{C B}=0.5 \mathrm{~V}$ and $V_{B E}=0.92 \mathrm{~V}$.

We have verified, using a bipolar-only technology integrating the main BiCMOS integration constraints, that circuits' specifications could indeed be met with these improved HBT performances. A frequency divider having a self-oscillation frequency of $77 \mathrm{GHz}$ (highest reported to date in any SiGe HBT/BiCMOS technology) and dividing correctly up to at least $100 \mathrm{GHz}$ has been demonstrated with a power consumption of only $122 \mathrm{~mW}$ [7]. The divider was tested over temperature and found to divide up to $97 \mathrm{GHz}$ at $50^{\circ} \mathrm{C}$ and up to $91 \mathrm{GHz}$ at $100^{\circ} \mathrm{C}$ (Fig. 7). A $105-\mathrm{GHz}$ VCO with a phase noise of $-101.3 \mathrm{dBc} / \mathrm{Hz}$ at $1 \mathrm{MHz}$ offset has been fabricated too [8]. It delivers $+2.7 \mathrm{dBm}$ of differential output power at $25^{\circ} \mathrm{C}$, with operation up to $125^{\circ} \mathrm{C}$. The measured spectrum of this record performance VCO is given in Fig. 8.

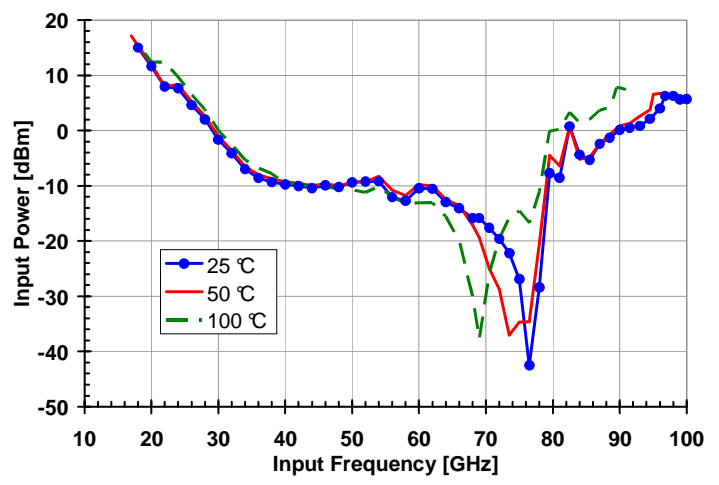

Fig. 7. Sensitivity curves across temperature for a $100 \mathrm{GHz}$ divider fabricated in FSA Si/SiGeC HBT technology [7].

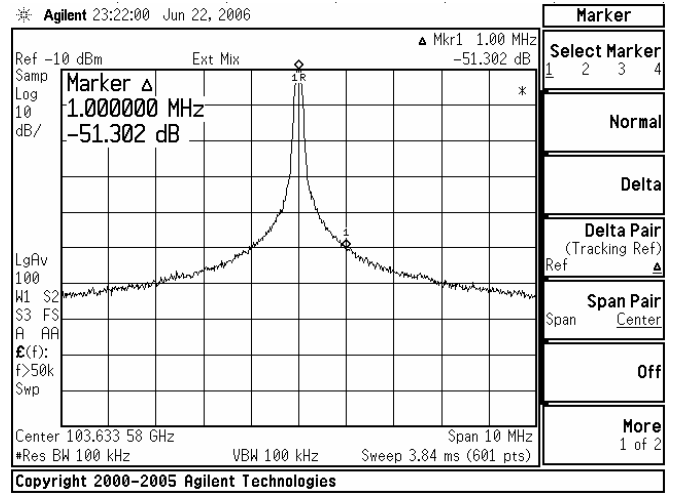

Fig. 8. Averaged spectral plot of phase noise (phase noise is $-101.3 \mathrm{dBc} / \mathrm{Hz}$ at $1 \mathrm{MHz}$ offset) of $105-\mathrm{GHz} \mathrm{VCO}$ in FSA $\mathrm{Si} / \mathrm{SiGeC}$ HBT technology [8].

The second limitation of BiCMOS9 is the back-end of line (BEOL). Indeed, all the previous results were obtained with a 6-copper metal layers digital CMOS BEOL, featuring 50- $\Omega$ microstrip (MS) transmission lines (TL) with a poor attenuation constant $\alpha$ of $1.2 \mathrm{~dB} / \mathrm{mm}$ at $80 \mathrm{GHz}$. BiCMOS9MW, whose development is under way, removes these two limitations since it embeds a 230$\mathrm{GHz} f_{T} / 280-\mathrm{GHz} f_{\max }$ HBT together with a BEOL dedicated to millimeter-wave applications, featuring $\alpha=$ $0.5 \mathrm{~dB} / \mathrm{mm}$ at $80 \mathrm{GHz}$ (cf. Fig. 9 and Table I). 


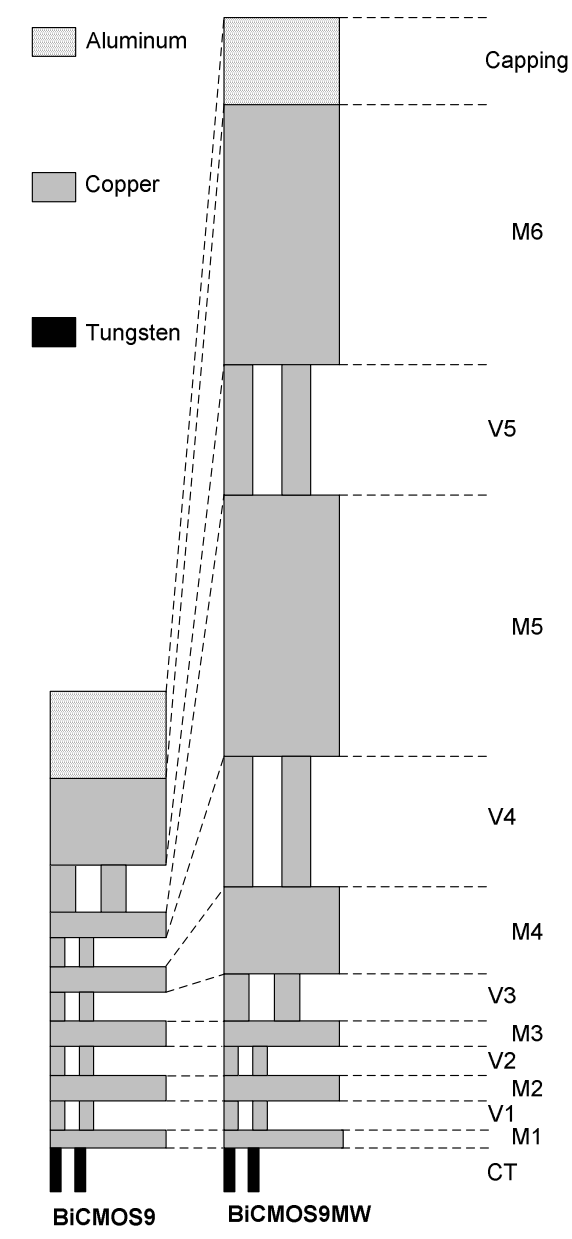

Fig. 9. Schematic comparison of the BEOL cross-sections of BiCMOS9 and BiCMOS9MW technologies (both feature 6 copper metal layers but with twofold height difference).

\section{END-OF-ROADMAP CHALLENGES}

\section{A. The BiCMOS roadmap}

BiCMOS roadmap does not follow Moore's law, whose driver is the shrink of digital functions. The move from one CMOS node to the next can be motivated by the increase of gate density. Then, HBT performance may remain unchanged if operation frequency of the targeted application does not change (e.g. cellular phone transceivers). This roadmap is likely to end with the dramatic increase of HF performances of advanced CMOS technologies.

On the contrary, high-speed BiCMOS roadmap is driven by, on one hand the increase of the optical communications data rate, and on the other hand the emergence of applications towards higher frequencies. The result of this is a step-by-step roadmap aiming at the best combination between CMOS density and HBT performance. This roadmap will go on as long as $\mathrm{Si} / \mathrm{SiGeC}$ HBT performances can be pushed forward (with significant advantages over CMOS) and applications to ever increasing frequencies carry on.

Advanced developments are in progress to further improve device characteristics, always having the BiCMOS integration as a target. Since HBT performance is not directly linked to a CMOS node, the improvement can be made inside a node (cf. BiCMOS9 / BiCMOS9MW) or from one node to another. Nevertheless, FEOL (front-end of line) and BEOL processing, overall thermal budget, and available design rules have an impact on the HBT performance through the vertical profile and/or the lateral dimensions. $f_{T}$ is indeed mainly determined by the vertical profile while lateral dimensions have considerable impact on $f_{\max }, N F_{\min }$ and power consumption.

\section{B. From $120 \mathrm{~nm}$ to $65 \mathrm{~nm} \ldots$ and $45 \mathrm{~nm}$ to the end of the roadmap}

From the current 120 -nm node to the next $90-\mathrm{nm}$ and 65-nm nodes, the main limitations are first, the final anneal performed for CMOS junctions activation $\left(\sim 1100^{\circ} \mathrm{C}\right.$ : only $\sim 30^{\circ} \mathrm{C}$ reduction in $65 \mathrm{~nm}$ compared to $120 \mathrm{~nm}$ and $90 \mathrm{~nm}$ ) and second, the BEOL design rules that are not in favor of the high current densities needed by the HBT. It is indeed important to notice that raising the collector doping to delay the onset of the Kirk effect, which is an efficient way to improve $f_{T}$, leads to an increase of the collector current density at peak $f_{T}$ (cf. Fig. 10). The limits of our present bipolar architecture, with the integration scheme in use (HBT between polygate deposition and final anneal), is probably close to $300-\mathrm{GHz} f_{T}$ for a CMOS spike anneal of $\sim 1100^{\circ} \mathrm{C}: 280-\mathrm{GHz} f_{T}$ and $300-\mathrm{GHz} f_{\max }$ has indeed been achieved while the extracted intrinsic cut-off frequencies of this device are $380-\mathrm{GHz} f_{T}$ and $350-\mathrm{GHz}$ $f_{\max }$ (cf. Fig. 11). Cryogenic measurements have shown that this architecture can potentially reach higher performances (at room temperature, with a different integration scheme) since $\sim 400-\mathrm{GHz} f_{T}$ and $440-\mathrm{GHz} f_{\max }$ have been measured at $50 \mathrm{~K}$ (cf. Fig. 12). This is the highest $f_{\max }$ ever reported for a SiGe:C HBT since the device reported in [12] features a relatively low $f_{\max }$ that does not increase significantly at low temperature. Circuit results have indeed shown that $f_{\max }$ must not be sacrificed for $f_{T}$ [7]. Architecture changes are currently being investigated to approach cryogenic performances at room temperature, without changing the integration scheme and keeping the $1100^{\circ} \mathrm{C}$ spike anneal. 


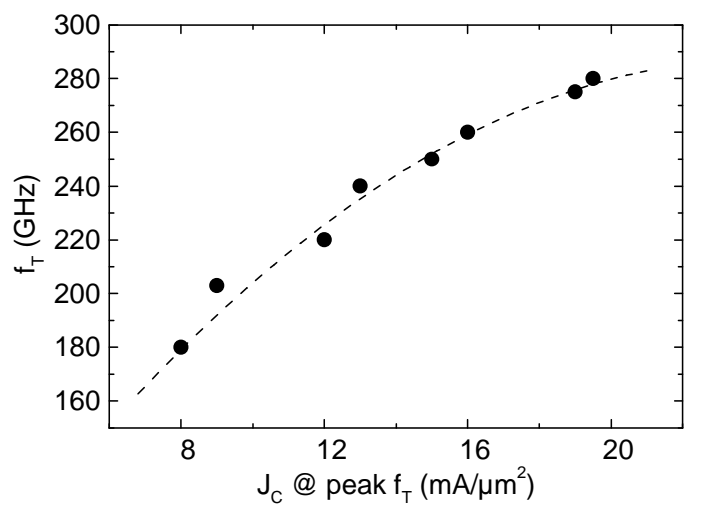

Fig. 10. Evolution trend of the current gain cut-off frequency $f_{T}$ with the collector current density $J_{C}$.

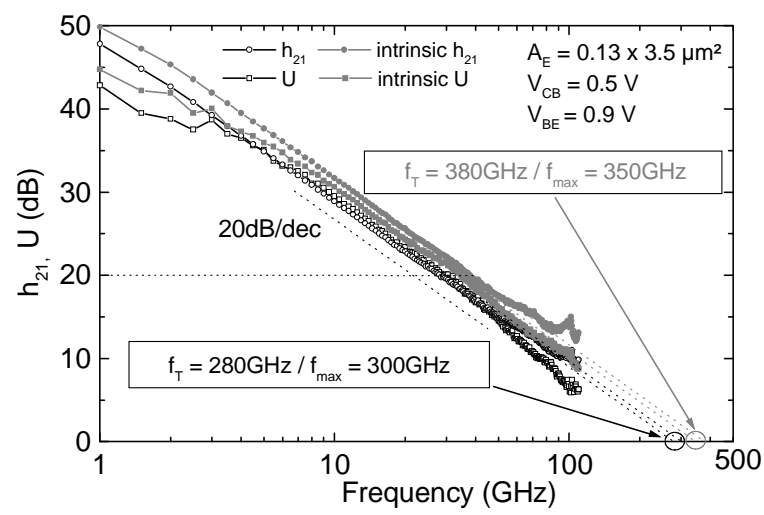

Fig. 11. HF gains vs. frequency characteristics of a $280-\mathrm{GHz} f_{T}$ - 300-GHz $f_{\max }$ HBT. Intrinsic device, extracted by removing extrinsic components, features $380-\mathrm{GHz} f_{T}$ and $350-\mathrm{GHz} f_{\max }$.

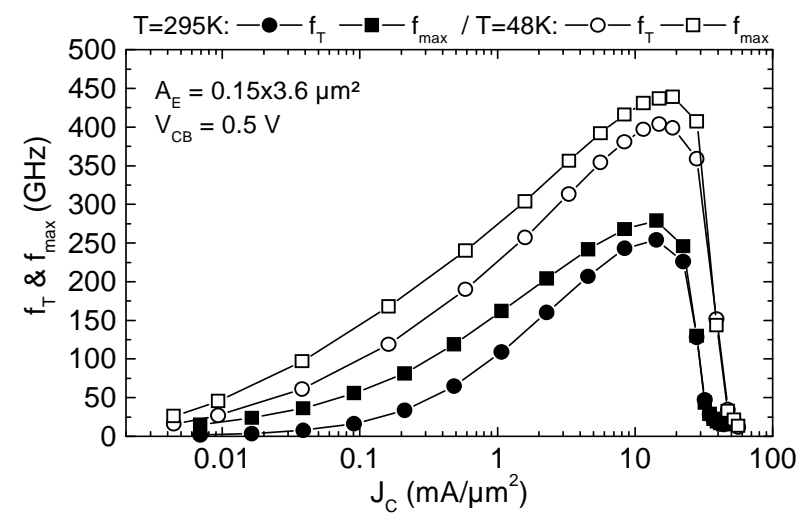

Fig. 12. $f_{T} \& f_{\max }$ vs. $J_{C}$ curves of a $\mathrm{Si} / \mathrm{SiGeC} \mathrm{HBT}$ at $295 \mathrm{~K}$ and $48 \mathrm{~K}$. Peak $f_{T}$ and $f_{\max }$ increase from $250 \mathrm{GHz} / 280 \mathrm{GHz}$ at $295 \mathrm{~K}$ to $400 \mathrm{GHz} / 440 \mathrm{GHz}$ at $48 \mathrm{~K}$.

From the 45-nm CMOS node to the end of the roadmap, the situation is reversed for the thermal budget since spike annealing is replaced by laser annealing, which allows dopant activation with nearly no diffusion. This is favorable for realizing narrow neutral base widths but calls for the development of new materials for the extrinsic base and for the emitter: For example, arsenic has to be replaced by phosphorus for the in-situ doping of the polyemitter. A TEM cross-section of a first device using a boron in-situ doped polybase, a phosphorus-doped emitter and nickel silicide is shown in Fig. 13. Beyond $45 \mathrm{~nm}$, the use of very thin SOI substrates that could belong to the mainstream CMOS will exacerbate the BiCMOS integration challenge [13].

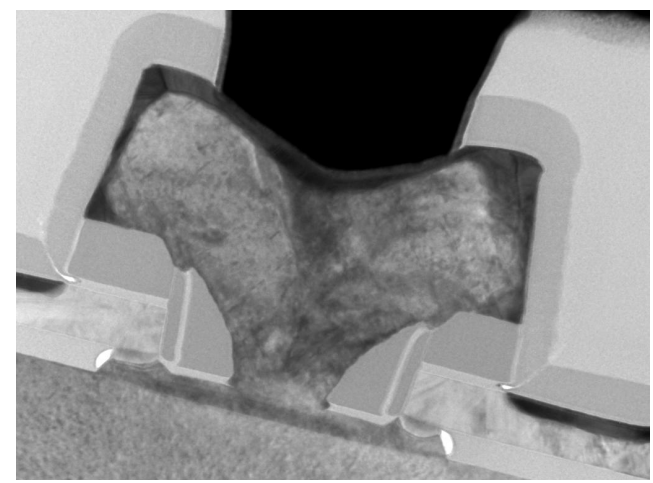

Fig. 13. TEM cross-section of a FSA Si/SiGeC HBT $\left(W_{E} \sim 100 \mathrm{~nm}\right)$ with boron in-situ doped polybase, phosphorus doped emitter and nickel silicide.

\section{Levering up the HBT limits}

Raising the transistor speed requests the increase of both the doping level of the neutral regions of the HBT and the abruptness of the junctions, which are conflicting targets. Tunneling currents may appear [11] due to this scaling and, more classically, $B V_{C E O}$ decreases for increasing $f_{T}$. Therefore, the main hurdle to maintaining a significant advantage over CMOS is to break up the classical tradeoff between $B V_{C E O}$ and $f_{T}$. A significant improvement has been achieved in the past years with the introduction of carbon in the base to reduce boron diffusion.

Solutions currently investigated to increase $B V_{C E O}$ without affecting $f_{T}$ focus on the increase on the base current [14]-[15]-[16]. Results of recent investigations, summarized in Fig. 14, have shown an encouraging progress of more than $50 \mathrm{GHz}$.V of the $f_{T} \times B V_{C E O}$ product by increasing the base current through base [15] or through emitter [16] engineering. This last solution, relying on a 'metallic' emitter transistor, can be obtained in a robust way by using an original process of poly replacement through contact holes (Fig. 15).

Nevertheless, high-power / high-frequency circuits should still require III-V technologies, in which breakdown voltages benefit from larger bandgap materials. 


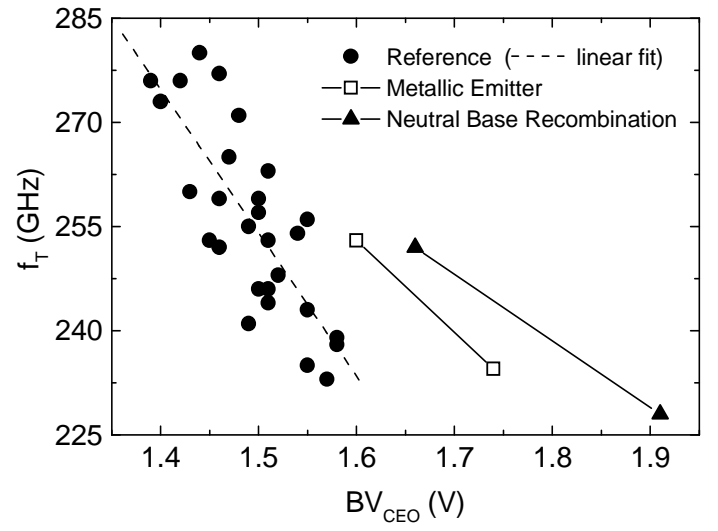

Fig. 14. $f_{T}-B V_{C E O}$ chart of different high-speed $\mathrm{Si} / \mathrm{SiGeC}$ HBTs: 'metallic emitter' and 'neutral base recombination' HBTs are compared to more standard devices (reference).

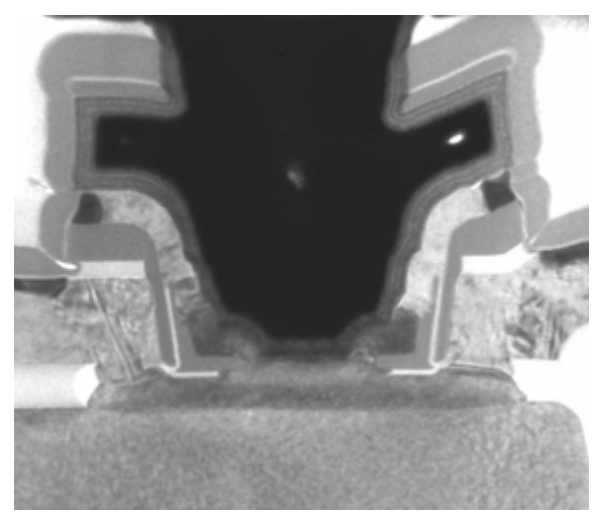

Fig. 15. TEM cross-section of a FSA Si/SiGeC HBT $\left(W_{E} \sim 150 \mathrm{~nm}\right)$ featuring a 'metallic emitter' obtained with a 'Poly REplacement Through Contact Holes' process.

\section{CONCLUSION}

First 120-nm SiGe BiCMOS platform from STMicroelectronics is released for production. This technology addresses applications up to $40 \mathrm{~Gb} / \mathrm{s}$ $40 \mathrm{GHz}$. Next BiCMOS platform will be able to cover applications up to $100 \mathrm{GHz}$. Efforts are underway to further increase SiGe HBT performances in spite of the constraints placed by the BiCMOS integration: no brick wall has been identified for the integration of high-speed HBTs in advanced CMOS nodes already defined. Nevertheless, the high-speed BiCMOS roadmap is not yet frozen since it depends on applications for which products are only in an advanced $R \& D$ phase.

\section{ACKNOWLEDGEMENT}

The authors wish to thank the staffs of the $200 \mathrm{~mm} \mathrm{Si}$ plants in STMicroelectronics Crolles and CEA-LETI Grenoble involved in the various aspects of this work.

\section{REFERENCES}

[1] P. Chevalier et al., "Advanced SiGe BiCMOS and CMOS platforms for Optical and Millimeter-Wave Integrated Circuits", in CSICS Tech. Dig., 2006, in press.

[2] M. Laurens et al., "A 150GHz fT / fmax 0.13 $\mu \mathrm{m} \mathrm{SiGe:C}$ BiCMOS technology”, in Proc. BCTM, 2003, pp 199-202.

[3] T.O. Dickson et al., "The Invariance of Characteristic Current Densities in Nanoscale MOSFETs and its Impact on Algorithmic Design Methodologies and Design Porting of Si(Ge) (Bi)CMOS High-Speed Building Blocks", IEEE J. Solid-State Circuits, vol. 41, no. 8, August 2006, pp. $1830-1845$.

[4] T.O. Dickson et al., "An 80-Gb/s 2^31 -1 Pseudo-Random Binary Sequence Generator in SiGe BiCMOS Technology", IEEE J. Solid-State Circuits, vol. 40, no.12, Dec. 2005, pp. 2735-2745.

[5] T.O. Dickson and S.P. Voinigescu, "Low-Power Circuits for a $2.5-\mathrm{V}, 10.7$-to- $86 \mathrm{~Gb} / \mathrm{s}$ Serial Transmitter in $130 \mathrm{~nm}$ SiGe BiCMOS", in CSICS Tech. Dig., 2006, in press.

[6] S. Pruvost et al., "A Compact Low Noise Amplifier in SiGe:C BiCMOS Technology for $40 \mathrm{GHz}$ Wireless Communications", in RFIC Symp. Dig., 2005, pp. 565-568.

[7] E. Laskin et al., "Low-Power, Low-Phase Noise SiGe HBT Static Frequency Divider Topologies up to $100 \mathrm{GHz}$ ", in Proc. BCTM, 2006, pp.xxxx.

[8] S.T. Nicolson et al., "Design and Scaling of SiGe BiCMOS VCOs Operating Near 100GHz", in Proc. BCTM, 2006, pp.xxxx.

[9] P. Chevalier et al., "230 GHz Self-Aligned SiGeC HBT for Optical and Millimeter-Wave Applications", IEEE J. SolidState Circuits, vol. 40, no. 10, Oct. 2005, pp. 2025-2034.

[10] P. Chevalier et al., " $300 \mathrm{GHz}$ fmax self-aligned SiGeC HBT optimized towards CMOS compatibility", in Proc. BCTM, 2005, pp. 120-123.

[11] D. Lagarde et al., "Band-to-band Tunneling in Vertically Scaled SiGe:C HBTs", IEEE Electron Dev. Lett., vol. 27 , no. 4, April 2006, pp. 275-277.

[12] R. Krithivasan et al., "Half-Terahertz Operation of $\mathrm{SiGe}$ HBTs", IEEE Electron Device Letters, vol. 27, no. 7, July 2006, pp. 567-569.

[13] P. Chevalier et al., "Low-Cost Self-Aligned SiGeC HBT Module for High-Performance Bulk and SOI RFCMOS Platforms", in IEDM Tech. Dig., 2005, pp. 983-986.

[14] B. Martinet et al., "An investigation of the static and dynamic characteristics of high speed SiGe:C HBTs using a poly-SiGe emitter", in Proc. BCTM, 2002, pp. 147-150.

[15] B. Barbalat et al., "Carbon effect on neutral base recombination in high-speed $\mathrm{SiGeC}$ HBTs", in Proc. ISTDM, 2006, pp. 238-239.

[16] B. Barbalat et al., "Experimental Study of Metallic Emitter SiGeC HBTs", in Proc. BCTM, 2006, pp.xxxx. 